Low molecular weight heparin induced thrombocytopenia and thrombosis. Eur J Haematol 1996;56:329-30.

4. Magnani HN. Heparin-induced thrombocytopenia (HIT): an overview of 230 patients treated with Orgaran (ORG 10172). Thromb Haemost 1993;70:554-61.

[Response declined]

12/8/83416

\section{Angiographic and electron-beam computed tomography studies of retrograde cardioplegia via the coronary sinus \\ To the Editor:}

We read with great interest the article "Angiographic and Electron-Beam Computed Tomography Studies of Retrograde Cardioplegia via the Coronary Sinus" authored by Farge and associates. ${ }^{1}$ Since 1993 our laboratory has been addressing the distribution of cardioplegic solution delivered via the coronary sinus using explanted human hearts. Many of the authors' findings are similar to our previously published results; however, there are significant differences that we believe are worthy of discussion.

Using their experimental technique, the authors could not demonstrate flow to the right ventricular free wall, regardless of whether the experimental contrast solution was delivered with the coronary sinus occluded at its ostium or not. In our article, "Gross and Microvascular Distribution of Retrograde Cardioplegia in Explanted Human Hearts," we found clear anatomic gross and microvascular histologic evidence for right ventricular free wall perfusion when cardioplegic solution was delivered with the coronary sinus occluded (Fig. 1). In that study we used an inert intracapillary marker (NTB-2) to qualitatively examine retrograde distribution. In another manuscript, "Coronary Sinus Ostial Occlusion During Retrograde Delivery of Cardioplegic Solution Significantly Improves Cardioplegic Distribution and Efficacy," also found clear evidence of capillary perfusion of right ventricular free wall myocardium. In this experiment colored microspheres were added to cardioplegic solution, which was delivered retrogradely with the coronary sinus either open or occluded, to quantitatively determine regional microvascular flow. Right ventricular flow, as well as posterior intraventricular septal flow, was greatly augmented as a result of coronary sinus occlusion. Even without occlusion, however, right ventricular flow was documented.

We believe these differing experimental conclusions are likely the result of differing experimental models. Flow through the coronary venous system and subsequent capillary or thebesian systems is governed by the resistance of the vascular beds and the viscosity of the fluid delivered. In our model, explanted human hearts are arrested by cold cardioplegia, excised and transferred to the laboratory, and subjected to experimental cardioplegic solutions within 20 minutes. We believe this model properly mimics the clinical situation, and normal operative coronary venous vascular resistance is anticipated. The authors use cadaveric hearts for experimentation, but they do not state how these are obtained and preserved and

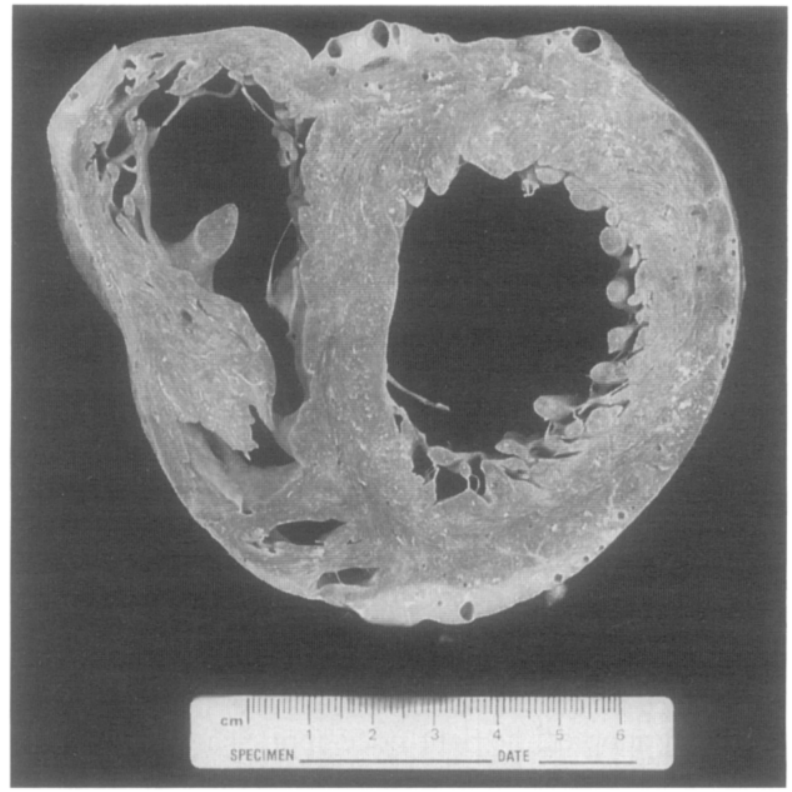

Fig. 1. Transverse section of an explanted human heart near the apex after coronary sinus perfusion of the inert intravascular marker NTB-2. Note that the material is seen in the vasculature of both the left and right ventricles.

within what time frame they are examined. Such conditions, particularly if ischemia is involved, can greatly alter vascular resistance. In our microsphere studies, the microspheres are delivered within human blood cardioplegic solution. In our anatomic NTB-2 studies, the experimental solution was prepared so that its $\mathrm{pH}$, viscosity, and osmolality all resembled blood cardioplegic solution. These properties of the authors' experimental solution are not mentioned within the article. Such variables, particularly viscosity, can significantly affect flow. We have found that to maintain a coronary sinus pressure of 30 to $40 \mathrm{~mm}$ $\mathrm{Hg}$, with the coronary sinus occluded, blood cardioplegic solution needs to be delivered at a rate of approximately $0.5 \mathrm{ml} / \mathrm{gm}$ of heart tissue per minute. The authors' finding of a coronary sinus pressure of $40 \mathrm{~mm} \mathrm{Hg}$ with a flow rate of $100 \mathrm{ml} / \mathrm{min}$ suggests either an unusually high vascular resistance or very small experimental hearts. Finally, when one considers the relative mass differences between the left and right ventricles, is it possible that some right ventricular perfusion is occurring during these experiments but that its identification is below the sensitivity of electron-beam computed tomography?

Despite our disagreement regarding right ventricular perfusion, this article contains two pieces of anatomic information that we have also found to be true. The first is the highly variable number of venovenous anastomoses between veins arising from the coronary sinus (most notably, the left anterior descending vein) and the posterior descending vein. We believe the quantity and quality of these anastomoses determine the amount of cardioplegic solution delivered to the posterior intraventricular septum and right ventricle when a transatrial coronary sinus cannula is being used, whether it is 
a manually inflating or self-inflating cannula. Like the authors, we too have found these connections to be extremely variable and have noted significant differences in right ventricular microvascular flow from one heart to another. As such, the surgeon who uses warm continuous transatrial retrograde cardioplegia will have consequent variable right ventricular protection. This is particularly true if cardioplegic flow rates are below 0.5 $\mathrm{ml} / \mathrm{gm}$ per minute. Indeed, our initial impetus for examining retrograde cardioplegic distribution was a poorly protected right ventricle after transatrial warm continuous retrograde blood cardioplegia. The second important finding in the article is the mean distance of just $1.7 \pm 0.6 \mathrm{~mm}$ between the coronary sinus ostium and the posterior descending vein. Some clinicians have suggested that by properly positioning a manually inflating retroperfusion cannula, one can improve cardioplegic distribution to the intraventricular septum and right ventricle by direct perfusion of the posterior descending vein. In most cases, this is an anatomic impossibility. Reliable perfusion of the posterior descending vein can be achieved only by direct cannulation of the coronary sinus through an outer purse-string suture or by the right atrial isolation approach to retrograde cardioplegia delivery.

Richard N. Gates, MD

Hillel Laks, $M D$

Davis C. Drinkwater, MD

Department of Surgery

Division of Cardiothoracic Surgery

UCLA School of Medicine

Los Angeles, CA 90095-1741

\section{REFERENCES}

1. Farge A, Mousseaux E, Acar C, et al. Angiographic and electron-beam computed tomography studies of retrograde cardioplegia via the coronary sinus. J Thorac Cardiovasc Surg 1996;112:1046-53.

2. Gates RN, Laks H, Drinkwater DC, et al. Gross and microvascular distribution of retrograde cardioplegia in explanted human hearts. Ann Thorac Surg 1993;56:410-7.

3. Rudis E, Gates RN, Laks $\mathrm{H}$, et al. Coronary sinus ostial occlusion during retrograde delivery of cardioplegic solution significantly improves cardioplegic distribution and efficacy. J Thorac Cardiovase Surg 1995;109:941-7.

[Response declined] 